University for Business and Technology in Kosovo

UBT Knowledge Center

UBT International Conference

2015 UBT International Conference

Nov 7th, 9:00 AM - 5:00 PM

\title{
Definition, Validation and Comparison of Two Population Models for Austria
}

\author{
Martin Bicher \\ Technische Universität Wien, martin.bicher@tuwien.ac.at \\ Barbara Glock \\ dwh simulation service, barbara.glock@dwh.at \\ Florian Miksch \\ dwh simulation service, florian.miksch@dwh.at \\ Niki Popper \\ Technische Universität Wien, niki.popper@tuwien.ac.at \\ Günter Schneckenreither \\ Technische Universität Wien, guenter.schneckenreither@tuwien.ac.at
}

Follow this and additional works at: https://knowledgecenter.ubt-uni.net/conference

Part of the Computer Sciences Commons, and the Digital Communications and Networking Commons

\section{Recommended Citation}

Bicher, Martin; Glock, Barbara; Miksch, Florian; Popper, Niki; and Schneckenreither, Günter, "Definition, Validation and Comparison of Two Population Models for Austria" (2015). UBT International Conference. 107.

https://knowledgecenter.ubt-uni.net/conference/2015/all-events/107

This Event is brought to you for free and open access by the Publication and Journals at UBT Knowledge Center. It has been accepted for inclusion in UBT International Conference by an authorized administrator of UBT Knowledge Center. For more information, please contact knowledge.center@ubt-uni.net. 
International Conference on Computer Science and Communication Engineering, Nov 2015

\title{
Definition, Validation and Comparison of Two Population Models for Austria
}

\author{
Martin Bicher ${ }^{1,2}$, Barbara Glock², Florian Miksch², Niki Popper ${ }^{1,2}$ \\ Günter Schneckenreither \\ ${ }^{1}$ Institute for Analysis and Scientific Computing, TU Wien, Wiedner Hauptstraße 8-10, \\ 1040 Vienna, Austria \\ ${ }^{2}$ dwh simulation service, dwh GmbH, Neustiftgasse 57-59, 1070 Vienna, Austria \\ \{martin.bicher, niki.popper, guenter.schneckenreither\}@tuwien,ac.at \\ \{barbara.glock, florian.miksch\}@dwh.at
}

\begin{abstract}
In this work we present two structurally different mathematical models for the prognostic simulation of Austria's population: A time-continuous, macroscopic system dynamics approach and a time-discrete, microscopic agent-based approach. Both models were developed as case studies of a series of population concepts in order to support models for decision-support in Austria's health care system. In the present work we want to focus on the definition, the parametrisation as well as especially the validation process of both population-models. The latter was of special interest as it included a cross-model validation with Statistics Austria's own prognostic model SIKURS.
\end{abstract}

Keywords: population model, model comparison, validation, cross-model validation

\section{Introduction}

As the patient always poses the centre of interest, valid prognostic modelling for decision support in the health care system is only possible if the underlying population is predicted validly as well. Doubtlessly long-term epidemiological or health-technology-assessment models (for example [5, 9]) can never be valid if the underlying population growth or decay is not considered.

Therefore developing models for predictive simulation of a country's population - so called population models - is one of the key tasks of health-care research project DEXHELPP. In order to create a valid founding for decision-support models for Austria's health care system a series of quality-assured population model concepts have been researched, developed and documented. In order to decide which of these concepts can be applied as a solid fundament of a decision-support model, two different (very basic) population models have been implemented and validated as well. These two case studies, a macroscopic system-dynamics model and a microscopic agent-based model, will be focus of this work. We will explain the two model concepts and finally focus on their interesting validation process.

\section{Modelling Approaches}

In this section we want to roughly describe the two used modelling methods and give a short definition of the developed models.

\subsection{System Dynamics and the System Dynamics Model}

System Dynamics. System Dynamics (short SD) is a macroscopic modelling technique developed by J.W. Forrester during the mid- 1950s [1, 2]. Herein a dynamic system is simulated using causal relationships between its components identified by the modeller. In case the causal relationships (also called flows) and their quantities are determined the temporal development of the system-components (also called stocks) are simulated by ordinary differential equations. 
System Dynamics Model. The model consists of 190 compartments, each of them representing the number of people of a certain age from 0 to $95+$ and their sex. Direct flows between them can be interpreted as ageing. Additional flows to sinks and from sources simulate births, deaths, and migration. The model was furthermore implemented using the simulation environment Any Logic [3].

\subsection{Agent-Based Modelling and the Agent Based Model}

Agent-Based Modelling. Compared to system dynamics, agent based modelling (short ABM) is a very young modelling technique as it became popular among modellers during the 1990s. It models individuals as agents with individual attributes and behavior. Classically these models are simulated with equidistant time-steps wherein each agent is addressed once to perform some behaviourcorresponding actions. As there is no definition of the modelling approach, all scientific fields agree with, the reader is referred to modelling guidelines presented at the 2006 Wintersimulation Conference [4] for more detailed information. Agent-Based Model. Each model agent represents one person in reality. Hence it is given a certain sex and age wherein the latter enhances with time. Additionally each agent has age- and sex-dependent probabilities to die and to emigrate. In case the agent is furthermore female, there is a probability to give birth to a child as well. Due to immigration, a number of individuals are additionally generated. The whole system is then simulated with time steps of arbitrary length (classically between 1 and 365 days). As Austria's population (about 8 Mio people) is unfortunately too big in order to simulate each individual at once, one model-agent is defined to pose for 100 persons. The model was moreover developed using the open source programming language Python 3 and executed with CPython.3.3.

\section{Parameterization and Validation}

\subsection{Concepts}

In order to correctly simulate the two models a specific research question is fixed: "Simulation of Austria's population from 01.01.2003 to 31.12.2050 with respect to age and sex". To reach this target both of the models first of all need to be correctly parameterised. Therefor datasets gained from Statistics Austria, probably Austria's biggest base for open-source socio-economic data and statistics [8], were used. Hereby the so called STATcube [7] provides a user friendly tool to search for data with almost arbitrary fineness and filters.

Altogether data-sets for migration, fertility and deaths for 2003-2014 were collected, each of them broken down to yearly age-categories and sex. Furthermore Statistics Austria applies the prognostic tool SIKURS [6] to create prognosis data up to the year 2076. Hence we additionally inquired prognostic data-sets according to the aforementioned real-data. As fertility prognoses were only available on the average, we developed a statistical extrapolation model to obtain this data for different age- categories as well. This data finally posed a firm basis for a direct parametrisation of both models. Hereby different time-scaling formulas have to be applied as the SD approach is time-continuous using transition-rates while the $\mathrm{ABM}$ is time-discrete with equidistant steps using transitionprobabilities. The target that the ABM should be valid for arbitrary length of the time-steps poses an additional challenge. As Statistics Austria additionally provides standard population data and prognosis also a basis for the validation-process of both models is provided: First, all models' results can be compared with collected population data for 2003-2014. Second, all type of cross-model validation can be performed comparing the models' results with prognostic data gained from the SIKURS tool via Statistics Austria. The total parametrisation and validation concept is visualized in Fig. 2. 


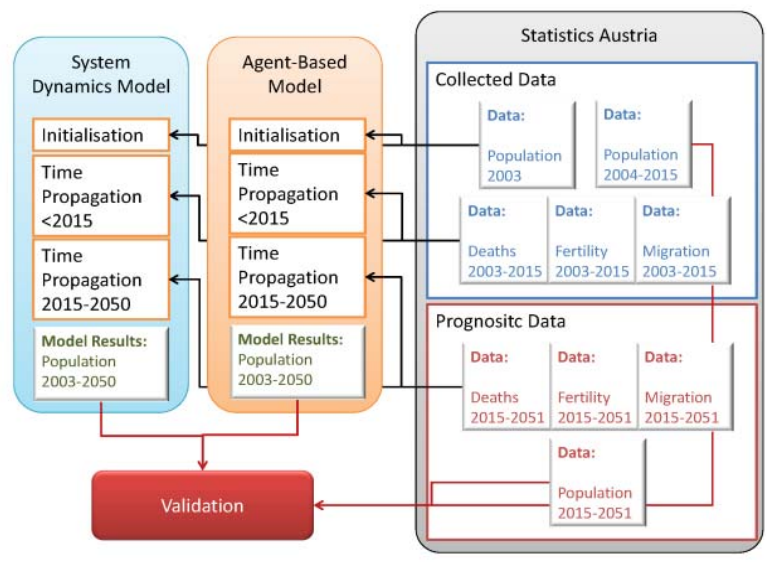

Fig. 2. Parametrisation- and validation-concept of both population models.

\subsection{Validation Results}

After brief verification and basic face-validation, finally, empirical validation was performed for both models at the same time. We decided to compare Statistics Austria (prognostic) data with the simulation results of the SD model and the ABM for two different time-step sizes (30 days and 365 days). As the ABM is a stochastic model,

50 simulation runs were executed and arithmetically averaged for the comparison.

Fig. 3 shows a direct comparison of the total population. Here we want to state a possible interpretation of three remarkable observations.

1. First, the SD results curve perfectly fits the prognosis curve of Statistics Austria. Our interpretation of this feature is that the prognostic SIKURS tool of Statistics Austria works quite similar to our system-dynamics model.

2. Second, it seems that the ABM with smaller step-size (30 days) notably overshoots the prognosis curve of Statistics Austria.

3. Third, this ABM simulation can be seen to perfectly fit the real-data between 2003 and 2015 while the SD model and the ABM with 365 day steps fail.

Thus, in terms of validity, we are facing the problem that the model producing better results in comparison with the real data reference, drifts off the reference for prognostic values.

Differences between different time-intervals of the ABM occur due to the parameterisation process respectively the parameter-calculation: Due to feedback reasons it is impossible to find an analytical formula to correctly scale the time-step- dependent transition-probabilities of the ABM. 


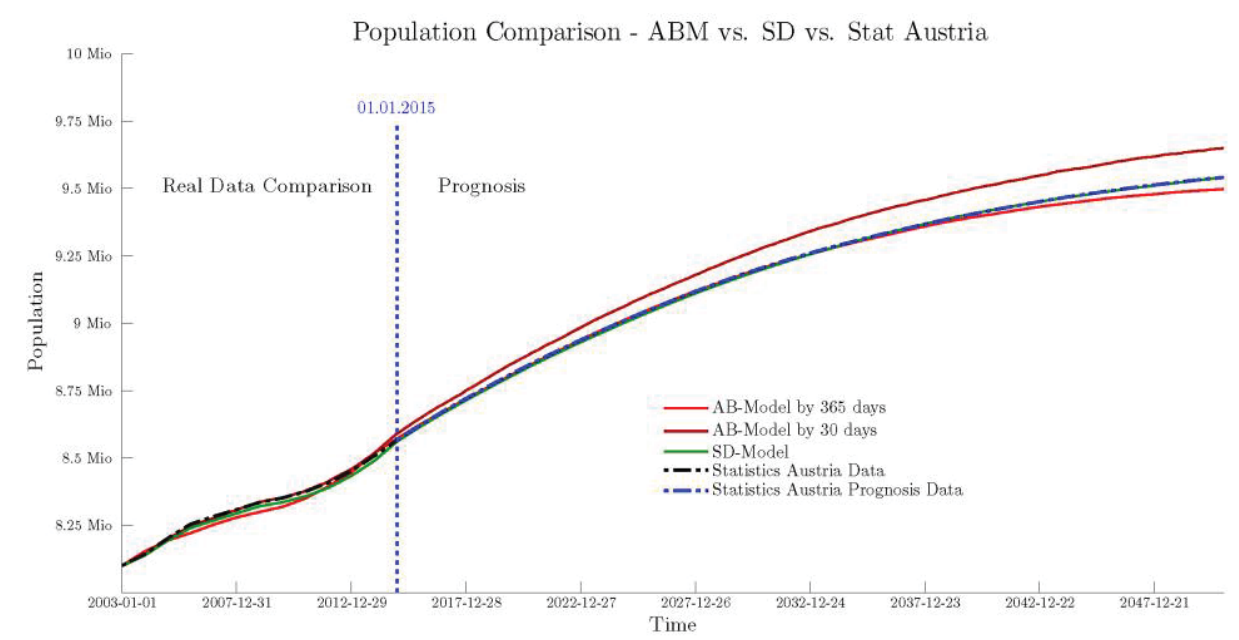

Fig. 3. Comparison of the total population for both models' results and Statistics Austria prognosis and collected data.

In addition to the empirical comparison of the total population several other tests were applied for both models each affirming the validity of both models. We furthermore want to lay special emphasis on the comparison of the age-structure of the population. Hereby we compared the age-pyramid for different points in time. The result can be seen in Fig. 4 on the example of 01.01.2015.

We see that the AB model perfectly fits the actual demographics of 2015. The SD model produces a smooth demographic curve, which is a natural artefact of SD models and can be considered as acceptable.

\section{Conclusion}

Both modelling approaches can be seen to be valid with respect to the data gained from Statistics Austria. In terms of different step-sizes, the agent-based approach leads to slightly different results. It finally depends on the availability and quality of data, which of them can be seen to be more correct. The SD approach perfectly matches the Statistics Austria prognosis for the aggregated numbers, but some flattening effect can be observed regarding demography. Furthermore the SD model stands out due to its fast computation time while the ABM provides a more flexible structure for extensions like regionality or socio-demographic factors. Summarising both models can be seen to validly simulate Austria's population and might be extended to simulate diseases and possible interventions. 

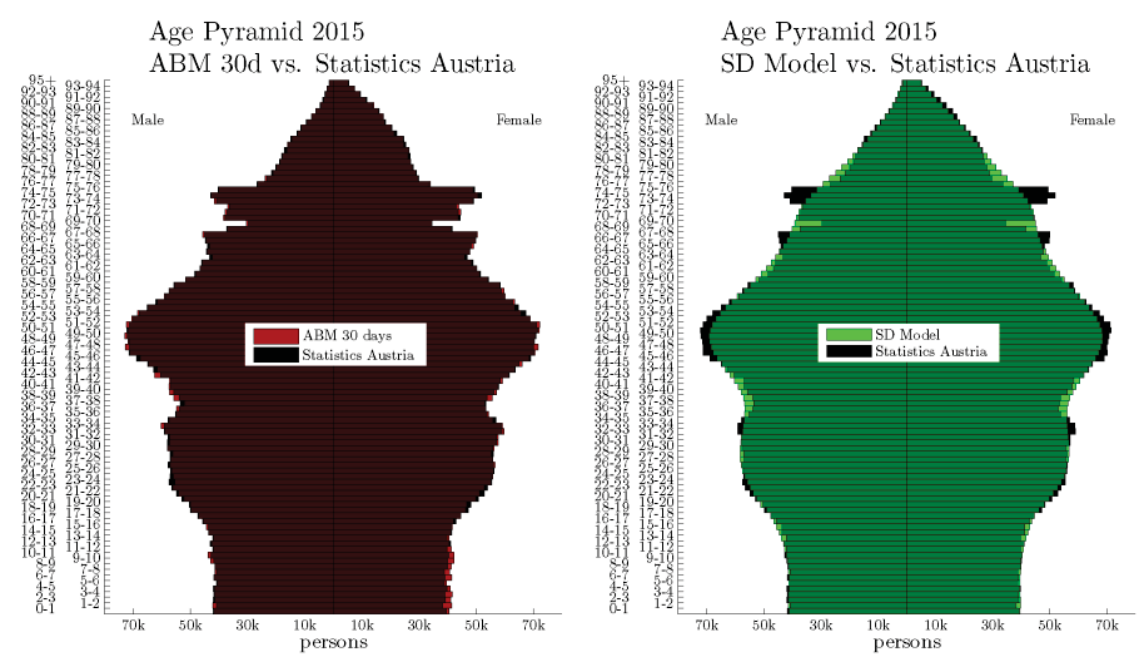

Fig. 4. A comparison between both models and Statistics Austria data from 01.01.2015 for different age-pyramids is shown.

Acknowledgments. K-Projekt DEXHELPP is supported by BMVIT, BMWFW and the state of Vienna via COMET - Competence Centers for Excellent Technolo-gies. Programme COMET is processed by FFG.

\section{References}

1. Forrester, J.W.: Industrial dynamics. Productivity Press, Cambridge, MA (1961).

2. Forrester, J.W.: World Dynamics. Cambridge, MA: Wright-Allen Press (1971).

3. Grigoryev, I.: AnyLogic 6 in three days: a quick course in simulation modeling. AnyLogic North America, Hampton, NJ (2012).

4. Macal, C.M., North, and M.J.: Tutorial on Agent-Based Modeling and Simulation Part 2: How to Model with Agents. In: Proceedings of the 2006 Winter Simulation Conference. pp. 73-

83, Monterey, California (2006).

5. Miksch, F. et al.: Modelling Spread of Pneumococcal Diseases in Austria: Long Term Behavior and Impact of Vaccination. In: Elst, G. (ed.) ASIM-Workshop 2009 in Dresden mit integrierter DASS'2009 - Workshop Beiträge. pp. 147-152 Fraunhofer IRB Verlag, Dresden, Germany (2009).

6. Statistik Austria: Demographisches Jahrbuch Österreich 2012. Verlag Österreich GmbH (2012).

7. Statistik Austria: StatCube,

http://www.statistik.at/web \_de/services/datenbank\_superstar/020627.html.

8. Statistik Austria: Statistik Austria, www.statistik.at.

9. Urach, C. et al.: Model selection process for cost effectiveness analysis of an organized

AAA screening in austria. In: ISPOR 15th Annual European congress. p. A473 (2012). 